\title{
OPORTUNIDADE EMPREENDEDORA OBSERVADA PELA LENTE DOS ASPECTOS COGNITIVOS
}

Simone Boruck Klein ${ }^{1}$

${ }^{1}$ FPL Fundação Pedro Leopoldo 


\section{OPORTUNIDADE EMPREENDEDORA OBSERVADA PELA LENTE DOS ASPECTOS COGNITIVOS}

Resumo: Este estudo tem como objetivo analisar a influência dos aspectos cognitivos na identificação e exploração das oportunidades empreendedoras. Verificou-se a relação do modelo mental empreendedor com esta tomada de decisão. Este artigo consiste num estudo teórico-empírico realizado através de um estudo comparativo de casos, com abordagem qualitativa. Buscou-se trazer da literatura internacional, por intermédio da abordagem psicológica os aspectos cognitivos fundamentais relacionados a oportunidade empreendedora. Foram entrevistados quatro empreendedores seriais e avaliou-se a relação existente entre os modelos mentais e a identificação e exploração de oportunidades. Como resultado da pesquisa, foi verificado que a identificação e exploração da oportunidade empreendedora é altamente relacionada aos dez aspectos cognitivos apurados, os achados corroboram totalmente em seis aspectos e parcialmente nos outros quatro, validando a premissa que norteou o estudo. Isto se apresenta relevante diante das questões que permeiam o estudo do processo empreendedor.

Palavras-chave: Empreendedorismo. Cognição Empreendedora. Oportunidades Empreendedoras. Modelos Mentais.

\section{Introdução}

O empreendedorismo tem se mostrado um excelente caminho para a busca do crescimento socioeconômico global, bem como fonte de inovação, abertura de novos mercados e empregabilidade (Van Praag e Versloot, 2007 ). Para se alcançar um alto nível de crescimento das atividades empreendedoras, por meio do fomento ao empreendedorismo, tem se revelado necessário compreender os fatores que tornam empreendedores bem sucedidos e as diferenças existente entre estes indivíduos e os demais (Behling, Lenzi, 2017).

Sabe-se que diferentes abordagens de pesquisas têm trazido crescimento no estudo deste fenômeno. Alguns caminhos percorridos por estas abordagens são de cunho econômico, por meio dos estudos seminais de Schumpeter (1972), outros com foco processual e ênfase na identificação de oportunidades, Shane e Venkataraman (2000), outros com abordagem organizacional, com foco no processo de criação de empresas, Gartner $(1989,2012)$ e a vertente da sociologia, preconizada por Weber (1958) que também fornece contribuições ao estudo deste fenômeno. Entretanto nesta pesquisa, a abordagem psicológica do empreendedor foi privilegiada devido ao recorte proposto pelo estudo.
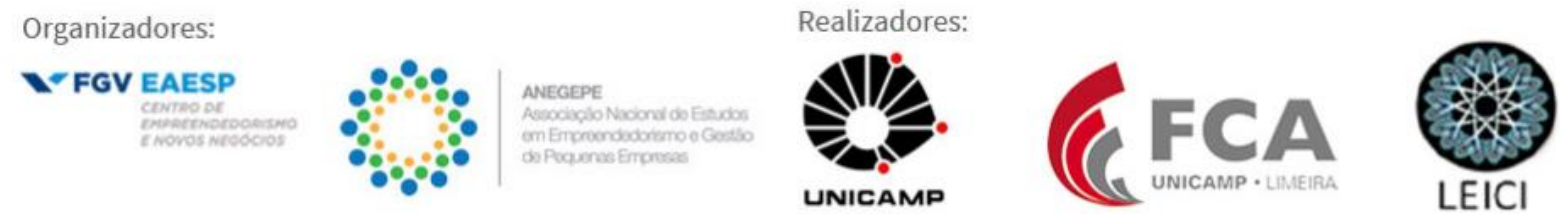
A abordagem psicológica teve como precursores Knight (1921), e com maior ênfase McClelland (1961). Esta abordagem privilegia identificar a figura do empreendedor, seu perfil, comportamento e personalidade. Seus estudos têm demonstrado algumas características do empreendedor como: motivação por conquistas e realizações, maior propensão em buscar desafios, ser o empreendedor um indivíduo diferente do não empreendedor (Gartner, 1989) e apresentar distinção de comportamento de gerentes em linha de frente (Filion, 1999). Atualmente, a abordagem psicológica tem voltado seus esforços para a identificação dos aspectos cognitivos mais relevantes para o empreendedorismo (Corbett, 2007).

Alguns modelos mentais que estão sendo delineados por meio de estudos na área, mostram que existem outros fatores importantes e determinantes para o sucesso do empreendedor. Estes fatores podem ser ambientais, sociais, emocionais, culturais entre outros, e afetam o desempenho do empreendedor em tomadas de decisão e busca de novas oportunidades. Entretanto, mesmo por meio de fontes de dados exógenos diversos, é a pessoa do empreendedor quem interpretará estes dados, portanto, seu modelo mental tem papel crucial na tomada de decisão. É ele quem dará significado aos dados e traçará um conjunto de ações empreendedoras na busca de resultados (Wood, McKelvie, Haynie, 2014). Busca-se neste estudo, um recorte intencional no aspecto da cognição empreendedora, onde será feito uma análise da influência de dez aspectos cognitivos na decisão de escolha de oportunidade de negócio.

A oportunidade empreendedora é vista neste estudo como a possibilidade de explorar um mercado de forma criativa e que gere valor ao empreendedor e ao mercado, sendo, portanto, sustentável. Entender como e por quem as oportunidades são descobertas, avaliadas e exploradas é de fundamental importância ao mundo dos estudos em empreendedorismo (Dimov, 2011; Krueger, 2003).

Este estudo teórico-empírico teve como objetivo fornecer suporte para argumentos teóricos anteriores sobre a cognição empreendedora e a busca de oportunidades, e usou a seguinte premissa para seu processo de investigação: Os aspectos cognitivos influenciam na identificação e exploração de oportunidades.

\section{Referencial Teórico}

\subsection{Empreendedorismo e a abordagem psicológica}

A abordagem psicológica dos estudos do empreendedorismo, é um campo que tem suas pesquisas em diversos caminhos contribuintes. Nos fluxos de estudos nesta área, as cognições relacionadas à tomada de decisão estão no ponto central. Na Figura 1, busca-se uma visualização holística de algumas abordagens, suas perspectivas e seus respectivos autores precursores dentro dos aspectos psicológicos do estudo do fenômeno do empreendedorismo.

\begin{tabular}{lll}
\hline Abordagem & Perspectiva & Autores Principais \\
\hline Lógica heurística & $\begin{array}{l}\text { Lógica baseada na simplificação } \\
\text { que os indivíduos utilizam para } \\
\text { tomar decisões, uso de atalhos }\end{array}$ & $\begin{array}{l}\text { Tversky e Kahneman(1973) } \\
\text { Busenitz e Barney(1997),Simon } \\
\text { e Houghton (2002), Baron } \\
\text { (2004) }\end{array}$ \\
& $\begin{array}{l}\text { Processos mentais únicos em } \\
\text { Estado de alertas e conexões }\end{array}$ & (1979), Kaish e Gilad
\end{tabular}


percebidas

Experiência empreendedora

Efetuação

Intenções, mecanismo cognitivos, infraestrutura cognitiva alertas para novas

oportunidades, saltos de lógica

Cognições empresariais desenvolvidas no empreendedor experiente, mentalidade empresarial mais consistente Pensamento e ação prosseguem uma tentativa de criar um dos vários possíveis resultados Ações empreendedoras
(1991), Gaglio e Katz (2001), Baron (2006), Busenitz e Arthurs (2007)

Mitchell et al. (2002), Baron e

Henry (2006), Ericsson, Krampe e Tesh-Romer (1993)

Sarasvathy (2001a) Sarasvathy $2001 \mathrm{~b}$ )

Baron (2006), Krueger Jr. (2000), Shepherd e Krueger (2002), McMullen e Shepherd (2006)

Figura 1: Diferentes abordagens cognitivas no estudo do fenômeno do Empreendedorismo Fonte: Adaptado de "The Central Question in Entrepreneurial Cognition Research 2007". Mitchell et al, 2007. P.6-9.

Conforme visto na Figura 1, as abordagens buscam resolver questões de avaliação, julgamento ou decisões relacionadas a oportunidade empreendedora. São diferentes caminhos que buscam a descoberta da "estrutura dos mecanismos cognitivos e suas interações com a base de conhecimento existente de um indivíduo" (Corbett, 2007, p. 98).

\subsection{Cognição Empreendedora e Oportunidades de Negócios}

Estudos da psicologia cognitiva partem de longa data e tem buscado significação em teorias e estudos empiricamente robustos. Como o fundamento do empreendedorismo é orientado pela busca de oportunidades (Stevenson e Jarillo, 2007), os estudos de cognição empreendedora têm buscado fundamentos na psicologia cognitiva.

A natureza da oportunidade pode ser definida como o esforço de criação em algo que o empreendedor acredita ter potencial para produzir riqueza (Hsieh e Kelley, 2016). E este reconhecimento, seria conexões entre pontos aparentemente não relacionados para alguns indivíduos, mas interligados para os outros (Baron, 2006). A geração de valor sobre uma oportunidade é feita de forma subjetiva e influenciada pelos modelos mentais intrínsecos de cada pessoa. Podem ser intuição, experiências prévias e capacidade de traçar paralelos de causa e efeito (Wood et al., 2014).

Ao estudar questões de oportunidades empreendedora, Mitchell et al. (2002) usou o termo cognição empreendedora como definição dos conhecimentos, habilidades e ações que o empreendedor toma na avaliação, no julgamento e na tomada de decisão sobre uma oportunidade de negócio. Tal identificação e avaliação das oportunidades requerem estudos personalizados devido a dependência intrínseca com a cognição individual (Shane e Venkataraman, 2000).

Os diferentes estilos cognitivos, ou modelos mentais, são determinantes e altamente influenciadores nas práticas das atividades organizacionais (Armstrong et al.,2012). Portanto, o estudo da cognição empreendedora procurar levantar questões de estruturas de conhecimentos necessários para uma avaliação e julgamento de oportunidades de empreendimentos, bem como compreender como os empreendedores utilizam modelos

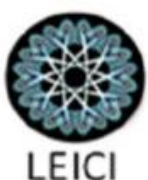


mentais simplificadores que agrupam informações permitindo a eles identificar oportunidades (Wood e Mckelvie, 2015; Busenitz et al, 2003).

\subsection{Modelos Mentais Empreendedores}

Os modelos mentais representam filtros que definem a forma como um indivíduo vê o mundo, são imagens internas arraigadas, que tanto limitam quanto expandem a forma de pensar e agir. Eles constituem representações simbólicas, por meio de modelos cognitivos, de como um indivíduo traduz e interpreta elementos e como projetam esses modelos para possíveis cenários futuros (Rook, 2013; Wood et al., 2014).

Em cenários complexos do mercado organizacional, tomar decisões corretas e rápidas, mesmo sem informações completas requerem do empreendedor modelos mentais eficazes. $\mathrm{O}$ modo de pensamento heurístico por exemplo, é baseado num modelo mental e é utilizado com frequência por empreendedores para simplificar decisões. Ele também é conhecido como vieses cognitivos, por utilizarem atalhos de processamento das informações e tem como fundamento a menor velocidade na avaliação e tomada de decisão e também a redução da incerteza (Shepherd, Willians e Patzelt, 2015). Outros modelos também estão sendo amplamente estudados e trazem contribuições teóricas de elementos comuns nos modelos mentais caracterizados como modelos mentais empreendedores.

Num estudo de ampla revisão bibliográfica internacional, Behling e Lenzi (2017) fazem um levantamento dos aspectos cognitivos inerentes ao empreendedor e que foram identificados mediante estudos teóricos-empíricos anteriores. Foram dez aspectos cognitivos considerados modeladores dos modelos mentais empreendedores relacionados a identificação e exploração de oportunidades levantados no estudo.

Os aspectos cognitivos predominantes para a identificação e exploração de oportunidades empreendedoras serão demonstrados na Figura 2, bem como a descrição e pesquisadores que comungam dos mesmos achados.

\begin{tabular}{lll}
\hline Aspectos Cognitivos & Descrição & Autores \\
\hline Experiência Prévia & A experiência prévia gera & Baron (2006), Sheperd, \\
& um conjunto de & Williams, Patzelt \\
& habilidades gerenciais e & (2015)Hsieh e Kelley \\
& técnicas, maior & (2016), Pryor et al (2016) \\
& sensibilidade para & Milan et al (2011) Krueger \\
& compreender mudanças e & Jr (2007) \\
& uma rede de contatos & \\
& Conhecimento acerca dos & Marvel, Davis, Sproul, \\
Domínio de Conhecimento & mercados, das & 2016), Alvarez e Barney \\
& necessidades e exigências & (2010), Shane (2000), \\
& dos clientes, e de aspectos & Wood, Mackelvie e \\
& técnicos & Haynie (2014) \\
& Experiências anteriores & Wood, Mackelvie, Haynie \\
tornam o empreendedor & (2014), Mitchell, Mitchell \\
& mais preparado, flexível e & e Smith (2008)
\end{tabular}


Propensão ao Risco

Excesso de Confiança

Otimismo

Criatividade

Capital Social

Reflexão Moral

Pensamento metacognitivo realista

Disposição em comprometer recursos significativos, de forma calculada, avaliação das condições de incerteza Superestimação das habilidades pessoais e da probabilidade de estar certo, ignorando novas informação

Tendência em esperar por resultados positivos, mesmo quando tais expectativas não são racionalmente justificadas

Capacidade de associar domínios até então desconectados, inovação Conexões externas do empreendedor e seus efeitos positivos

Orientação por valores pessoais em situações nas quais objetivos pessoais conflitam com interesses sociais

Processo pelo qual ele(a) reflete e conscientiza-se sobre o leque de estratégias disponíveis e apropriadas
Baldacchino et al. (2015),

Hsieh e Kelley (2015),

Shepherd, Williams,

Patzelt (2015)

De Carolis, Saparito

(2006), Sánchez, Carballo, Gutiérrez (2011), Sheperd, Williams, Patzelt (2015), Hogarth e Karelaia (2012)

Shepherd, Williams, Patzelt (2015), Sánchez, Carballo, Gutiérrez (2011), Cassar (2010), Ucbasaran et al. (2010), Hmieleski e

Baron (2009)

Martin, Wilson (2016), Fills, Rentschler (2010), Ward (2004)

De Carolis, Saparito (2006), Marvel, Davis, Sproul (2016), Yang e Zhang (2015)

Shepherd, Williams, Patzelt (2015), Vignochi, Lezana, Camilotti (2013), Bryant (2009), Milan et al. (2011)

Haynie, Shepherd (2009), Krueger Jr (2007), Sheperd, Williams e Patzelt (2015)

Figura 2: Aspectos cognitivos dos modelos mentais de empreendedores que influenciam a identificação de exploração de oportunidades

Fonte: Adaptado de "Oportunidades empreendedoras e modelos mentais: aspectos cognitivos que influenciam a exploração de oportunidades. Behling, G., e Lenzi, F. C. 2017,p. 3-12.

\section{Procedimentos metodológicos}

Este estudo foi realizado utilizando o estudo comparativo de casos, onde foi usada uma abordagem qualitativa, descritiva e aplicada (Yin, 1984; Cooper e Schindler, 2006). Busca-se por intermédio deste método identificar e analisar as relações entre aspectos cognitivos e a identificação de oportunidades. Segundo Gaglio e Katz (2001) para estudos

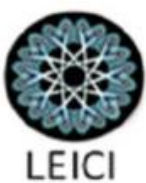


relacionados a oportunidades empreendedoras os métodos qualitativos são apropriados por levar o indivíduo a pensar, ao contrário de somente recordar-se das experiências passadas.

As unidades de análise constituem-se dos aspectos cognitivos dos empreendedores selecionados bem como as unidades de observação são os próprios empreendedores. $\mathrm{O}$ critério de escolha foi intencional e motivado pelas características pessoais dos pesquisados, por serem empreendedores em série, que segundo Wright, Robbie e Ennew (1997), são aqueles que se envolvem na abertura de várias empresas. Utilizou-se para a coleta de dados a técnica de entrevista em profundidade e semiestruturada. As entrevistas foram baseadas nas variáveis que emergiram da revisão bibliográfica, e compuseram 33 questões. Foram adotados os 10 aspectos cognitivos levantados pela literatura por Behling e Lenzi (2017) e expostos na Figura 2, sendo formulada três perguntas respectivamente para cada um dos aspectos cognitivos relacionados. Também uma questão referente aos setores de negócios onde houveram as identificações e explorações das oportunidades e duas referentes a dados demográficos e área de formação. A coleta de dados ocorreu presencialmente com duração aproximada de 55 minutos para cada empreendedor. A premissa que orientou este processo de pesquisa foi: Os aspectos cognitivos influenciam na identificação de oportunidades.

A análise dos dados ocorreu pelo método de análise de conteúdo, e foram utilizados os critérios defendidos por Gil ( 2008), que são: redução, exibição e a revisão, todos aplicados aos dados para buscar padrões, regularidades e discrepâncias. As limitações da pesquisa referem-se à regionalidade, todos os empreendedores são da mesma região do país. A partir deste estudo pretende-se trazer contribuições à teoria e não a generalização dos resultados.

Como forma de validação externa, buscou-se utilizar os procedimentos aplicados por Eisenhardt (1989) para análise dos dados. Foram confrontados os dados obtidos na pesquisa com os principais fundamentos teóricos e empíricos existentes na busca de confirmá-los, complementá-los ou contradizê-los.

\section{Análise da Influência dos Dez Aspectos Cognitivos na Identificação e Exploração das Oportunidades}

Foram entrevistados 4 empreendedores com perfil de empreendedores serial, ou seja, que já participaram da abertura de diversas organizações bem como da análise da viabilidade das oportunidades destas empresas. Serão denominados de E.1, E.2, E.3 e E.4. devido ao acordo de confidencialidade e de proposta somente para questões acadêmicas. A Figura 3 traz informações demográficas, área de formação e número de empresas fundadas pelos empreendedores.

\begin{tabular}{lllll}
\hline Entrevistados & Gênero & Idade & $\begin{array}{l}\mathrm{N}^{\circ} \text { Empresas } \\
\text { Fundadas }\end{array}$ & $\begin{array}{l}\text { Área de Formação/ } \\
\text { Especialização }\end{array}$ \\
\hline E.1 & $\mathrm{M}$ & 49 anos & 4 & Economia \\
E.2 & $\mathrm{F}$ & 43 anos & 5 & Administração \\
E.3 & $\mathrm{M}$ & 52 anos & 3 & Nutrição \\
E.4 & $\mathrm{F}$ & 35 anos & 3 & Marketing \\
\hline
\end{tabular}

Figura 3: Dados dos Entrevistados

Fonte: Dados da pesquisa
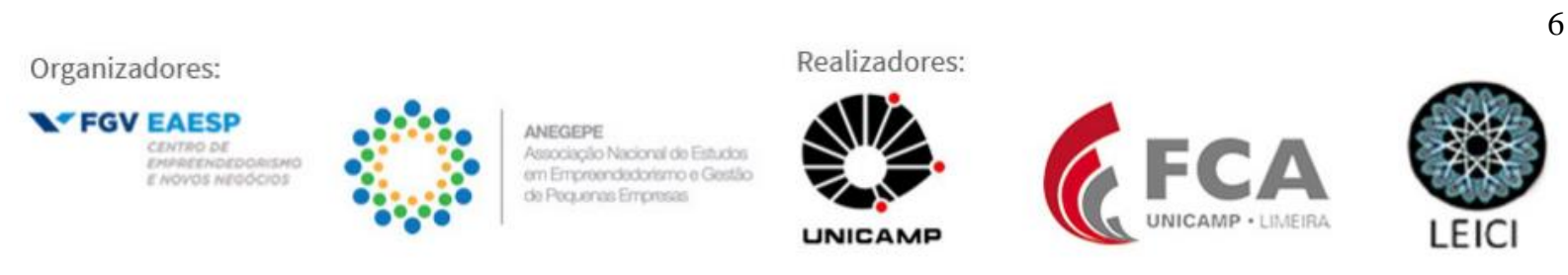
Quanto as características das empresas fundadas pelos entrevistados, a Figura 4 mostra os segmentos de atuação de cada empresa e se havia sócios em cada empreendimento. Foi denominado como "S" para composição societária e "I" individual.

\begin{tabular}{lllll}
\hline $\begin{array}{l}\text { Entrevistado/ } \\
\text { Empreendimento }\end{array}$ & E.1 & E.2 & E.3 & E.4 \\
\hline Empreendimento 1 & Loja varejo/S & Prest.serviço/S & Prest.serviço/S & Loja varejo/I \\
Empreendimento 2 & Distribuidora/I & Prest.serviço/I & Loja varejo/I & Loja varejo/I \\
Empreendimento 3 & Indústria/S & Loja varejo/I & Restaurante/I & $\begin{array}{l}\text { Lanches } \\
\text { Gourmet/ S }\end{array}$ \\
Empreendimento 4 & Indústria/S & Loja varejo/I & & \\
Empreendimento 5 & & Prest.serviço/S & & \\
\hline
\end{tabular}

Figura 4: Segmento de atuação das empresas fundadas e suas composições: societária ou individual

Fonte: Dados da pesquisa

Quanto aos resultados da entrevista o E.1 apresentou aspectos cognitivos razoavelmente favoráveis em relação a experiência prévia, pois antes de empreender já havia trabalhado em três diferentes organizações, duas no segmento de vendas, porém em outro ramo de atividade. Quanto ao domínio do conhecimento, no seu primeiro empreendimento, loja de varejo, ele realizou cursos específicos sobre segmento e conhecia parcialmente as necessidades dos clientes, já que o segmento era prematuro no Brasil. No segundo empreendimento tinha domínio quase total de conhecimento pois era no mesmo segmento da loja de varejo. No terceiro empreendimento não tinha domínio do conhecimento do mercado, onde seu sócio tinha este requisito. Viu como excelente oportunidade por se tratar de um mercado de consumo de massa o qual obteve insucesso. No quarto empreendimento não tinha domínio do conhecimento, e da mesma maneira confiou no conhecimento do sócio, viu a oportunidade por se tratar de uma indústria com mark-up alto.

No terceiro item, referente ao fracasso anterior, o primeiro, segundo e terceiro empreendimento não tinha esta experiência, porém, seu terceiro empreendimento teve insucesso, sendo este aspecto "fracasso anterior" fato real na abertura do quarto empreendimento. No quarto item, propensão ao risco, o primeiro e o segundo empreendimento apresentaram baixo propensão a investimentos, já nos dois últimos apresentou alta propensão. No quinto item, excesso de confiança, ele se identificou como altamente detentor em todos os quatro investimentos, muitas vezes ignorando novas informações importantes sobre a oportunidade. No sexto item, otimismo, o E.1 foi classificado como altamente otimista em todas as oportunidades empreendidas. No sétimo item, criatividade, foi classificado parcialmente detentor desta característica. No oitavo item, capital social, o primeiro e o segundo empreendimento ele contou com alto grau desta capacitação, já no terceiro e no quarto investimento, não compunha o capital social adequado para o setor. No nono item, reflexão moral, foi considerado razoavelmente favorável a
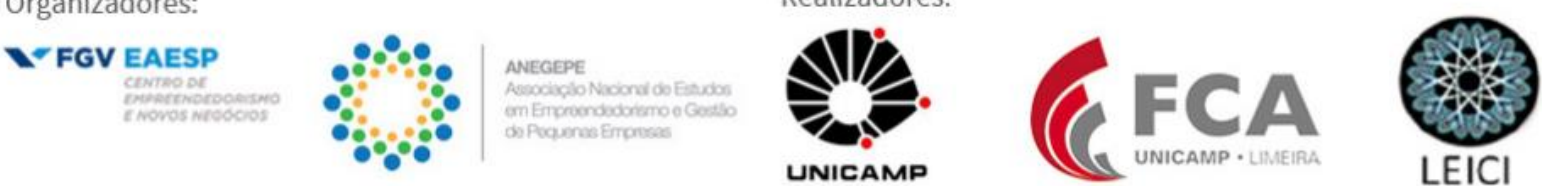
reflexão quanto aos interesses sociais, e no décimo item, pensamento metacognitivo, apresentou alta cognição neste aspecto ao avaliar as oportunidades de negócio.

O E.2 apresentou os seguintes resultados: altamente favorável em relação a experiência prévia, pois antes de abrir sua empresa de prestação de serviços já trabalhava como funcionária em uma empresa do mesmo setor. No segundo, terceiro, quarto e quinto empreendimento não tinha experiência prévia no setor. Quanto ao domínio do conhecimento, em todos os cinco empreendimentos considerava-se conhecedora do mercado e das necessidades dos clientes, além de aspectos técnicos da área. No quesito ao fracasso anterior, as experiências de insucesso do segundo e quarto empreendimentos foram considerados altamente importantes pela entrevistada, na propensão ao risco, somente no quarto investimento a empreendedora se considerou ousada, nos restantes tinha pouca disposição em comprometer recursos. Quanto ao excesso de confiança, ela apresentou alto grau de confiabilidade em si em todas as oportunidades de negócios, no quesito otimismo, apresentou resultado parcialmente favorável, sentindo-se levemente pessimistas em alguns casos. No item criatividade, considera-se altamente inovadora, porém razoavelmente criativa, quanto ao capital social, nos empreendimentos 1,2,3, apresentava alto grau de conexões externas no setor, já nos empreendimentos 4 e 5 seu capital social era menos denso. No aspecto reflexão moral, pensa ter crescido nos últimos investimentos, estando cada vez mais orientada pelos interesses sociais. No aspecto metacognitivo apresentou razoável relação nos primeiros 2 empreendimentos, e altamente favorável nos 3 últimos empreendimentos.

Para o E.3 a experiência prévia o ajudou, pois, antes de abrir seu primeiro empreendimento já havia trabalhado em diversas empresas, tendo habilidades técnicas de atendimento ao cliente, trabalho em equipe entre outras. $\mathrm{O}$ domínio do conhecimento foi altamente favorável em seus negócios, pois buscou mentores que pudessem o auxiliar nos conhecimentos que não dominava. Quanto ao fracasso anterior, sente-se mais preparado pois no primeiro empreendimento não obteve sucesso completo, e isto lhe trouxe experiência. No item propensão ao risco, vê-se como altamente disposto a comprometer recursos significativos, tem ousadia e sente-se destemido. No quesito excesso de confiança, sente-se altamente confiante em sua intuição, muitas vezes ignorando informações relevantes. Quanto ao otimismo, percebe ter diminuído com o passar do tempo e diante das incertezas do mercado. Na criatividade, percebe-se menos propenso, com baixa propensão a esta característica, no capital social, em seu primeiro empreendimento não obtinha as conexões externas suficientes, no segundo e terceiro empreendimento houve alta propensão ao capital social. Na reflexão moral, pensa sempre ter buscado interesses sociais parcialmente, e no item pensamento metacognitivo, demonstrou ter razoável pensamento estratégico.

O E.4 contou com experiência prévia, pois seu pai era grande empreendedor e ela acompanhou seus empreendimentos. No domínio do conhecimento, os dois primeiros empreendimentos eram relacionados a sua "grande paixão" (hobby) o que ajudou na busca das oportunidades e do conhecimento. Quanto ao fracasso anterior, considera que as experiências de insucesso do pai contribuíram muito para seu aprendizado. Na propensão ao risco, houve um decréscimo de sua disposição em investir, no primeiro empreendimento agiu com maior propensão e nos dois segundos com maior cautela. Quanto ao excesso de confiança, considera também ser no primeiro empreendimento muito confiante, ignorando informações importantes, e nos últimos dois empreendimentos menos confiante. No otimismo, apontou

Organizadores:

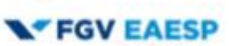

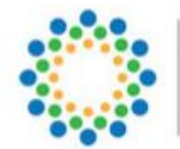

AMEGBE

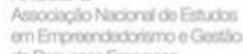
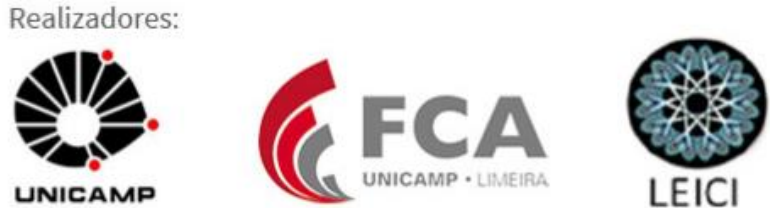
alto grau, "entrando de cabeça" como ela mesmo relatou. Quanto a criatividade, mostrou-se altamente criativa e inovadora. No item capital social, apresentou razoável acesso em todos os empreendimentos. Na reflexão moral, apresentou-se menos propensa aos interesses sociais, e no pensamento metacognitivo, mostrou ter cognição altamente estratégica.

A definição da relação cognitiva empreendedora dos entrevistados segundo suas respostas é relacionada na Figura 5 em relação a identificação e exploração de oportunidades em cada empreendimento realizado por eles, no E.1, quatro empreendimentos E.2 cinco empreendimentos, E.3 três empreendimentos e E.4 três empreendimentos. Os principais resultados são apontados de forma esquematizada em "A" para altamente relacionado, "R" para razoavelmente relacionado e "P" para pouco relacionado.

\begin{tabular}{lcccc}
\hline Aspectos Cognitivos & E.1 & E.2 & E.3 & E.4 \\
\hline Experiência Prévia & RAAA & AARAR & AAA & AAA \\
Domínio de Conhecimento & AAAA & AAAAA & AAA & AAR \\
Fracasso Anterior & PPPA & PRAAA & PAA & AAA \\
Propensão ao Risco & RRRR & PPPAP & AAA & ARP \\
Excesso de Confiança & AAAA & AAAA & AAA & AAA \\
Otimismo & AAAA & AARAR & ARP & ARR \\
Criatividade & RRRA & RAAAA & RRP & AAA \\
Capital Social & AAPP & AAAPR & AAA & RRR \\
Reflexão Moral & AAAA & PPRRA & RRR & PRR \\
Pensamento & AAAA & RAAA & AAA & AAA \\
Metacognitivo & & & & \\
\hline
\end{tabular}

Figura 5: Resultados dos aspectos cognitivos empreendedores voltados para identificação e exploração de oportunidades.

Fonte: Elaboração própria com base nos resultados da pesquisa, 2018.

\section{Discussão dos Resultados}

Após a análise comparativa entre os empreendedores entrevistados foi possível verificar alguns dados importantes. Dentre os entrevistados, foi unanime para todas as identificações e explorações de oportunidades o aspecto cognitivo "excesso de confiança". Também se destacam os aspectos cognitivos: pensamento metacognitivo e domínio do conhecimento, ambos com alto grande de compatibilidade entre os empreendedores. A reflexão moral apresentou crescimento para todos os entrevistados, mostrando nesta amostra, que quanto mais se empreendeu e o tempo passou, mais propensão existiu a atenção aos interesses comuns. A propensão a risco apresentou índices razoáveis de relevância, mostrando que o empreendedor nem sempre está disposto a comprometer recursos significativos e de forma incalculada. $\mathrm{O}$ capital social também apresentou uma tendência a se tornar menos relevante ao olhar do empreendedor em cada novo empreendimento, talvez pelo excesso de confiança intrínseco ao empreendedor.

Após a análise dos dados foi possível verificar que a premissa que direcionou este estudo foi integralmente validada, pois verificou-se que os aspectos cognitivos influenciam na identificação de oportunidades, devido aos resultados altamente relacionados com teoria que o embasou. Na Figura 6, faz-se a validação externa da pesquisa, comparando-a com estudos anteriores sobre o tema.

\section{Organizadores:}

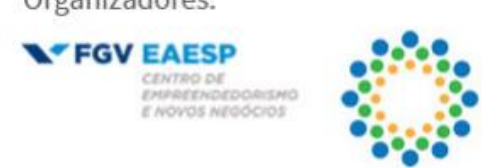

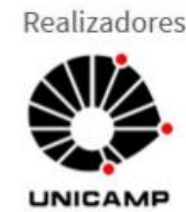

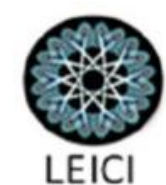




\begin{tabular}{|c|c|c|}
\hline Aspectos cognitivos & $\begin{array}{l}\text { Resultado } \\
\text { estudo }\end{array}$ & Marco teórico \\
\hline Experiência prévia & $\begin{array}{c}\text { Corrobora os } \\
\text { anteriores }\end{array}$ & $\begin{array}{l}\text { Baron (2006), Sheperd, } \\
\text { Williams, Patzelt } \\
\text { (2015)Hsieh e Kelley (2016), } \\
\text { Pryor et al (2016) Milan et al } \\
\text { (2011) Krueger Jr (2007) }\end{array}$ \\
\hline Domínio do conhecimento & $\begin{array}{c}\text { Corrobora os } \\
\text { anteriores }\end{array}$ & $\begin{array}{l}\text { Marvel, Davis, Sproul, 2016), } \\
\text { Alvarez e Barney (2010), } \\
\text { Shane (2000), Wood, } \\
\text { Mackelvie e Haynie (2014) }\end{array}$ \\
\hline Fracasso anterior & $\begin{array}{c}\text { Corrobora os } \\
\text { anteriores }\end{array}$ & $\begin{array}{l}\text { Wood, Mackelvie, Haynie } \\
\text { (2014), Mitchell, Mitchell } \\
\text { Smith (2008) }\end{array}$ \\
\hline Propensão ao risco & $\begin{array}{c}\text { Confirma } \\
\text { parcialmente }\end{array}$ & $\begin{array}{l}\text { Baldacchino et al. (2015), } \\
\text { Hsieh e Kelley (2016), Yang } \\
\text { e Zhang (2015), Shepherd, } \\
\text { Williams, Patzelt (2015) }\end{array}$ \\
\hline Excesso de confiança & $\begin{array}{c}\text { Corrobora os } \\
\text { anteriores }\end{array}$ & $\begin{array}{l}\text { De Carolis, Saparito (2006), } \\
\text { Sánchez, Carballo, Gutiérrez } \\
\text { (2011), Sheperd, Williams, } \\
\text { Patzelt (2015), Hogarth e } \\
\text { Karelaia (2012) }\end{array}$ \\
\hline Otimismo & $\begin{array}{c}\text { Corrobora os } \\
\text { anteriores }\end{array}$ & $\begin{array}{l}\text { Shepherd, Williams, Patzel } \\
(2015) \text {, Sánchez, Carballo, } \\
\text { Gutiérrez (2011), Cassar } \\
\text { (2010), Ucbasaran et al. } \\
(2010) \text {, Hmieleski e Baron } \\
(2009)\end{array}$ \\
\hline Criatividade & $\begin{array}{c}\text { Confirma } \\
\text { parcialmente }\end{array}$ & $\begin{array}{l}\text { Martin, Wilson (2016), Fills, } \\
\text { Rentschler } \quad \text { (2010), Ward } \\
(2004)\end{array}$ \\
\hline Capital social & $\begin{array}{c}\text { Confirma } \\
\text { parcialmente }\end{array}$ & $\begin{array}{l}\text { De Carolis, Saparito (2006), } \\
\text { Marvel, Davis, Sproul (2016), } \\
\text { Yang e Zhang (2015) }\end{array}$ \\
\hline Reflexão moral & $\begin{array}{c}\text { Confirma } \\
\text { parcialmente }\end{array}$ & $\begin{array}{l}\text { Shepherd, Williams, Patzel } \\
\text { (2015), Vignochi, Lezana, } \\
\text { Camilotti (2013), Bryant } \\
\text { (2009), Milan et al. (2011) }\end{array}$ \\
\hline Pensamento metacognitivo & $\begin{array}{c}\text { Corrobora os } \\
\text { anteriores }\end{array}$ & $\begin{array}{l}\text { Haynie, Shepherd (2009), } \\
\text { Krueger Jr (2007), Sheperd, } \\
\text { Williams e Patzelt (2015) }\end{array}$ \\
\hline
\end{tabular}

Figura 6: Análise dos resultados da pesquisa com a teoria

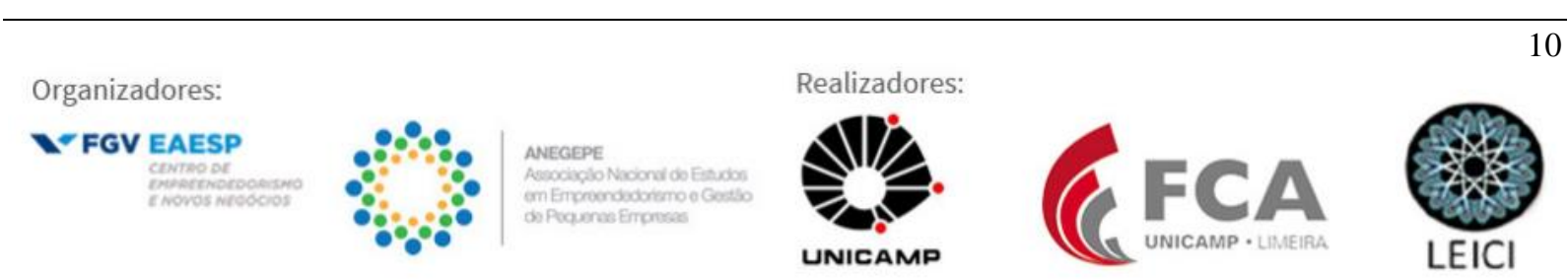


Fonte: Elaboração própria com base nos resultados da pesquisa, 2018.

\section{Considerações finais}

Por intermédio da abordagem psicológica, este artigo buscou trazer da literatura internacional os aspectos cognitivos que têm se destacado em estudos relacionados ao processo empreendedor, mais especificamente o processo de identificação e exploração de oportunidades. Este estudo se justifica por ser o campo de estudos do empreendedorismo grandemente categorizado em por que, quando e como oportunidades de criação de novos negócios relevantes surgem em uma economia (Venkataraman, 1997). Entender este processo é de fundamental importância para saber como fomentar deste fenômeno. Após levantar os aspectos cognitivos na literatura, foi aplicado de forma empírica um estudo de caso múltiplo com empreendedores seriais, ou seja, que já se envolveram na abertura de mais de um empreendimento. Buscou-se com isto contribuir para a validação externa da literatura.

Como resultado da pesquisa, foi verificado que a identificação e exploração de oportunidades é altamente relacionado aos aspectos cognitivos já relacionados em estudos anteriores. Isto demonstra como os modelos mentais tem alta influência nas oportunidades empreendedoras. Os aspectos cognitivos: experiência prévia, domínio do conhecimento, fracasso anterior, excesso de confiança, otimismo e pensamento metacognitivo validaram os estudos anteriores. Já os aspectos cognitivos: propensão ao risco, criatividade, capital social e reflexão moral tiveram uma relação menos intensa em nosso estudo, onde confirmaram parcialmente os resultados anteriores.

Sabe-se que aspectos afetivos têm grande influência nos modelos mentais, porém estes não foram pesquisados devido ao recorte do estudo em se concentrar nos aspectos cognitivos. Isto apresenta-se como delimitação da pesquisa, bem como, a regionalidade pois todos os entrevistados são da mesma região do país.

Como lacuna de pesquisa identificada, verificou-se que o estudo de como a formação do conhecimento é adquirida e transformada nas cognições do empreendedor trará um avanço no caminho para descoberta do processo empreendedor integral. Poderá contribuindo no processo de ensino, na formação de equipes empreendedoras como também para os gerentes de recursos humanos tomarem decisões em relação ao desenvolvimento de suas equipes.

\section{Referências}

Alvarez, S. A. e Barney, J. B. (2010). Entrepreneurship and epistemology: The philosophical underpinnings of the study of entrepreneurial opportunities. Academy of Management annals, 4(1), 557-583.

Armstrong, S. J., Cools, E. e Sadler-Smith, E. (2012). Role of cognitive styles in business and management: Reviewing 40 years of research. International Journal of Management Reviews, 14(3), 238-262.

Baldacchino, L., Ucbasaran, D., Cabantous, L.e Lockett, A. (2015). Entrepreneurship research on intuition: a critical analysis and research agenda. International Journal of Management Reviews, 17(2), 212-231.

Baron, R. (2004). The cognitive perspective: A valuable tool for answering entrepreneurship's basic "why" questions. Journal of Business Venturing, 19, 221-239.
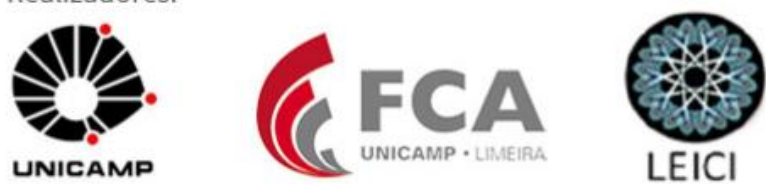
Baron, R. A. (2006). Opportunity recognition as pattern recognition: How entrepreneurs "connect the dots" to identify new business opportunities. The Academy of Management Perspectives, 20(1), 104-119.

Baron, R. e Henry, R. (2006). The role of expert performance in entrepreneurship: how entrepreneurs acquire the capacity to excel. In Babson Conference Proceedings.

Behling, G.e Lenzi, F. C. (2017). Oportunidades empreendedoras e modelos mentais: aspectos cognitivos que influenciam a exploração de oportunidades. XLI Encontro da Anpad,São Paulo, SP, Brasil.

Bryant, P. (2009). Self-regulation and moral awareness among entrepreneurs. Journal of Business Venturing, 24(5), 505-518.

Busenitz, L. W. e Barney, J. B. (1997). Differences between entrepreneurs and managers in large organizations: Biases and heuristics in strategic decision-making. Journal of business venturing, 12(1), 9-30.

Busenitz, L. W., West III, G. P., Shepherd, D., Nelson, T., Chandler, G. N. e Zacharakis, A. (2003). Entrepreneurship research in emergence: Past trends and future directions. Journal of management, 29(3), 285-308.

Busenitz, L. W. e Arthurs, J. D. (2007). Cognition and capabilities in entrepreneurial ventures. The psychology of entrepreneurship, 131-150.

Cooper, D. R., Schindler, P. S. e Sun, J. (2006). Business research methods (Vol. 9). New York: McGraw-Hill Irwin.

Corbett, A. C. e Hmieleski, K. M. (2007). The conflicting cognitions of corporate entrepreneurs. Entrepreneurship Theory and Practice, 31(1), 103-121.

De Carolis, D. M. e Saparito, P. (2006). Social capital, cognition, and entrepreneurial opportunities: A theoretical framework. Entrepreneurship theory and practice, 30(1), 41-56.

Dimov, D. (2011). Grappling with the unbearable elusiveness of entrepreneurial opportunities. Entrepreneurship Theory and Practice, 35(1), 57-81.

Eisenhardt, K. M. (1989). Building theories from case study research. Academy of management review, 14(4), 532-550.

Ericsson, K.A., Krampe, R.T. e Tesch-Romer, C. (1993). The role of deliberate practice in the acquisition of expert performance. Psychological Review, 100, 363-406.

Filion, L. J. (1999). Empreendedorismo: empreendedores e proprietários-gerentes de pequenos negócios. Revista de administração, 34(2), 5-28.

Fillis, I. e Rentschler, R. (2010). The role of creativity in entrepreneurship. Journal of Enterprising Culture, 18(01), 49-81.

Gaglio, C.M. e Katz, J.A. (2001). The psychological basis of opportunity identification: Entrepreneurial alertness. Small Business Economics, 16(2), 95-111.

Gartner, W. B. (1989). Some suggestions for research on entrepreneurial traits and characteristics. Entrepreneurship theory and practice, 14(1), 27-38.

Gil, A. C. (2008). Métodos e técnicas de pesquisa social. 6. ed. Ediitora Atlas SA.

Haynie, J. M., Shepherd, D. A., \& McMullen, J. S. (2009). An opportunity for me? The role of resources in opportunity evaluation decisions. Journal of Management studies, 46(3), 337361.

Hmieleski, K. M. e Baron, R. A. (2009). Entrepreneurs' optimism and new venture performance: A social cognitive perspective. Academy of management Journal, 52(3), 473-488. 
Hogarth, R. M., \& Karelaia, N. (2012). Entrepreneurial success and failure: Confidence and fallible judgment. Organization Science, 23(6), 1733-1747.

Hsieh, Ru-Mei, Kelley, D. J. (2016). The role of cognition and information access in the recognition of innovative opportunities. Journal of Small Business Management, v.5,4,n. S1, p. 297-311.

Gartner, W. B. (2012). Entrepreneurship as organization creation. Handbook on organizational entrepreneurship, 21-30.

Kaish, S. e Gilad, B. (1991). Characteristics of opportunities search of entrepreneurs versus executives: Sources, interests, general alertness. Journal of business venturing, 6(1), 45-61.

Kirzner, I. M. (1979). Perception, opportunity, and profit. University.

Knight, F. H. (1921). Risk, uncertainty and profit. New York: Hart, Schaffner and Marx.

Krueger Jr, N. F., Reilly, M. D., e Carsrud, A. L. (2000). Competing models of entrepreneurial intentions. Journal of business venturing, 15(5-6), 411-432.

Krueger Jr., N. F. (2003). The cognitive psychology of entrepreneurship. In Handbook of entrepreneurship research(pp. 105-140). Springer, Boston, MA.

Krueger Jr., N. F. (2007). The cognitive infrastructure of opportunity emergence. In Entrepreneurship (pp. 185-206). Springer, Berlin, Heidelberg.

Martin, L.; Wilson, N. (2016). Opportunity, discovery and creativity: A critical realist perspective. International Small Business Journal, 34(3), 261-275.

Marvel, M. R., Davis, J. L., e Sproul, C. R. (2016). Human capital and entrepreneurship research: A critical review and future directions. Entrepreneurship Theory and Practice, 40(3), 599626.

McClelland, D. C. (1961). The achievement society. Princenton, NJ: Von Nostrand.

McMullen, J. S.e Shepherd, D. A. (2006). Entrepreneurial action and the role of uncertainty in the theory of the entrepreneur. Academy of Management review, 31(1), 132-152.

Milan, G. S., De Toni, D., Dorion, E., e Schuler, M. (2011). A influência dos modelos mentais dos empreendedores no desempenho de suas organizações. GESTÃO. Org-Revista Eletrônica de Gestão Organizacional-ISSN: 1679-1827, 8(3).

Mitchell, R. K., Busenitz, L., Lant, T., McDougall, P. P., Morse, E. A., e Smith, J. B. (2002). Toward a theory of entrepreneurial cognition: Rethinking the people side of entrepreneurship research. Entrepreneurship theory and practice, 27(2), 93-104.

Mitchell, R. K., Busenitz, L. W., Bird, B., Marie Gaglio, C., McMullen, J. S., Morse, E. A.,e Smith, J. B. (2007). The central question in entrepreneurial cognition research 2007. Entrepreneurship theory and practice, 31(1), 1-27.

Mitchell, R. K., Mitchell, J. R., e Smith, J. B. (2008). Inside opportunity formation: Enterprise failure, cognition, and the creation of opportunities. Strategic Entrepreneurship Journal, 2(3), 225-242.

Pryor, C., Webb, J. W., Ireland, R. D., \& Ketchen Jr, D. J. (2016). Toward an integration of the behavioral and cognitive influences on the entrepreneurship process. Strategic Entrepreneurship Journal, 10(1), 21-42.

Rook, L. (2013). Mental models: a robust definition. The Learning Organization, 20(1), 38-47.

Sánchez, J. C., Carballo, T., e Gutiérrez, A. (2011). The entrepreneur from a cognitive approach. Psicothema, 23(3). 
Sarasvathy, S.D. (2001a). Causation and effectuation: Toward a theoretical shift from economic inevitability to entrepreneurial contingency. Academy of Management Review, 26(2), 243288.

Sarasvathy, S.D. (2001b). Entrepreneurship as economics with imagination. Business Ethics Quarterly. The Ruffin Series, 3, 95-112.

Schumpeter, J. A. (1972). History of economic analysis. Ed. from manuscript by Elizabeth Boody Schumpeter (No. 330.15/S39h).

Shane, S. (2000). Prior knowledge and the discovery of entrepreneurial opportunities. Organization science, 11(4), 448-469.

Shane, S., e Venkataraman, S. (2000). The promise of entrepreneurship as a field of research. Academy of management review, 25(1), 217-226.

Shepherd, D. A., e Krueger, N. F. (2002). An intentions-based model of entrepreneurial teams' social cognition. Entrepreneurship Theory and Practice, 27(2), 167-185.

Shepherd, D. A., Williams, T. A., e Patzelt, H. (2015). Thinking about entrepreneurial decision making: Review and research agenda. Journal of management, 41(1), 11-46.

Simon, M., e Houghton, S. M. (2002). The relationship among biases, misperceptions, and the introduction of pioneering products: Examining differences in venture decision contexts. Entrepreneurship Theory and Practice, 27(2), 105-124.

Stevenson, H. H., e Jarillo, J. C. (2007). A paradigm of entrepreneurship: Entrepreneurial management. In Entrepreneurship (pp. 155-170). Springer, Berlin, Heidelberg.

Tversky, A., e Kahneman, D. (1973). Availability: A heuristic for judging frequency and probability. Cognitive psychology, 5(2), 207-232.

Ucbasaran, D., Westhead, P., Wright, M., e Flores, M. (2010). The nature of entrepreneurial experience, business failure and comparative optimism. Journal of business venturing, 25(6), 541-555.

Van Praag, C. M., e Versloot, P. H. (2007). What is the value of entrepreneurship? A review of recent research. Small business economics, 29(4), 351-382.

Vignochi, L., Rojas Lezana, Á. G., e Camilotti, L. (2013). Empreendedorismo e valores humanos: um estudo conceitual. Gestão. Org: Revista Eletrônica de Gestão Organizacional, 11(2).

Ward, T. B. (2004). Cognition, creativity, and entrepreneurship. Journal of business venturing, 19(2), 173-188.

Weber, M. (1958). The Protestant Ethic and the Spirit of Capitalism: The relationships between religion and the economic and social life in modern culture. Anthony Giddens, trans. Talcott Parsons (New York: Charles Scribner's Sons, 1976).

Wood, M. S., McKelvie, A., e Haynie, J. M. (2014). Making it personal: Opportunity individuation and the shaping of opportunity beliefs. Journal of Business Venturing, 29(2), 252-272.

Wood, M. S., e McKelvie, A. (2015). Opportunity evaluation as future focused cognition: Identifying conceptual themes and empirical trends. International Journal of Management Reviews, 17(2), 256-277.

Wright, M., Robbie, K., e Ennew, C. (1997). Serial Entrepreuneurs. British Journal of Management, 8 (3), 251-268.

Yang, J., e Zhang, J. (2015). Social networks, cognition and risk recognition in new ventures: Evidence from China. Journal of Developmental Entrepreneurship, 20(02), 1550012.
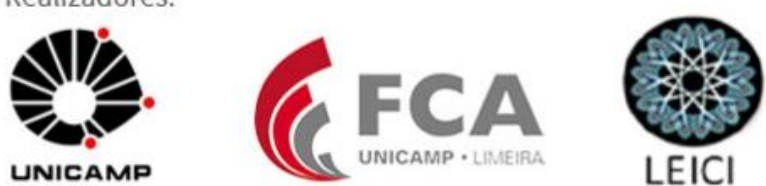


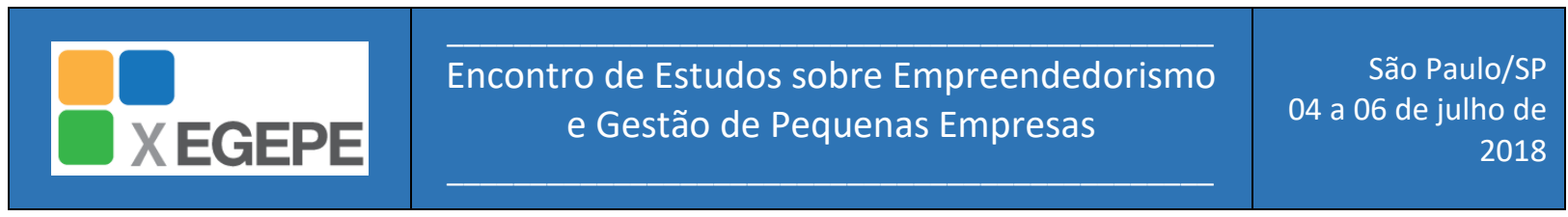

Yin, R. (1984). Case study research 5th Edition. Beverly Hills: Sage Publications. 